\title{
Sen y algunos escritos en la constitución del campo del Desarrollo Humano (Bibliografía anotada de Amartya Sen)
}

\author{
Gardy Augusto Bolívar Espinoza*
}

Resumen: Se presenta un breve y sintético recorrido de la producción teórica publicada de Amartya Sen y algunos textos clásicos que acompañan la fundación del campo del desarrollo Humano. La intención es mostrar el proceso de su pensamiento y la diversidad de temas que plantea. El supuesto es que Sen expresa sintéticamente la complejidad del campo.

Palabras clave: Bibliografía de Sen, el campo del Desarrollo Humano.

\section{Sen and some writings in the constitution of the field of Human Development (Amartya Sen's writen Bibliography)}

\begin{abstract}
A brief and synthetic route of the published theoretical production of Amartya Sen and some classic texts that accompany the foundation by the field of the Human development are hereby presented. The intention is to show the process of her thought and the diversity of subjects she raises. The assumption is that Sen synthetically expresses the complexity of the field.
\end{abstract}

Key words: Sen’s Bibliography , the field of Human Development.

Recibida: 25.06.2009

Aceptada: 15.07.2009

$* * *$

\section{Presentación}

Amartya Sen, nace en Shantiniketan, India, en 1933. Estudió en India y luego se licenció y doctoró en Trinity College de Cambridge. Profesor de economía en universidades de Calcuta, Delhi, Oxford y Harvard, desde enero del 2004, permanece en Harvard. Durante largos años fue reconocido entre sus pares, como el principal merecedor al Premio Nobel de Economía, el cual, lo obtiene en 1998 «por sus contribuciones a la economía del bienestar» y por haber "ayudado a restaurar la dimensión ética del debate económico y social, combinando herramientas económicas y

\footnotetext{
* Universidad Autónoma Metropolitana, Unidad Azcapotzalco, Ciudad de México, México. Email: abe88@hotmail.com
} 
filosóficas. También, además de múltiples reconocimientos y premios, fue galardonado con el Bharat Ratna en 1999 por su trabajo en el campo de la matemática económica.

Sen es uno de los pocos economistas modernos muy respetado y reconocido desde todas las esquinas del espectro intelectual. El periódico The Observer lo ha llamado "la madre Teresa de la economía", pero independientemente de la broma reveladora, se le reconoce ampliamente y muy seriamente la "muy, muy fuerte fibra moral que sostiene toda su obra y la auténtica preocupación por el hombre y su dignidad”.

Tanto por su amplitud y calidad, por la difusión de su obra y por el ambiente intelectual que le ha rodead,o Amartya Sen puede ser considerado como el símbolo de la investigación sobre el Desarrollo Humano. Él atrae un conjunto de influencias que sobrepasan su rico aporte intelectual. Los aportes que otros investigadores han hecho -ya sea en disputa o acuerdo con él — han configurado el vasto y complejo campo agonístico que, no sin reparos, puede ser denominado Desarrollo Humano: imbricación de ética y economía, filosofías, políticas públicas, y ciencias sociales en general con una vocación humanista contemporánea.

Se ha distinguido por sus trabajos sobre la comunicación social, las hambrunas, los mecanismos subyacentes de la pobreza y su medición; últimamente, por sus trabajos sobre identidad y violencia y en muchos otros más. En el campo de la «Economía del Bienestar» se ha distinguido por su profundidad y seriedad, e incluso se afirma en algunos círculos que la "Economía del Bienestar" es casi un sinónimo de Amartya Sen; lo mismo se podría decir respecto del estudio de las hambrunas, la pobreza y el desarrollo Humano.

\section{La fundación del campo agonístico}

Amartya Sen se forma en un ambiente plural de grandes tradiciones que con diferente intensidad nutren su pensamiento y ayudan a conformar el campo del Desarrollo humano. Campo que se diseña bajo la sombra tutelar intelectual y moral de John Maynard Keynes, colaborador de Marshall. La obra clásica de este último The General Theory of Employment, Interest and Money (Keynes, 1936) permite una prolífera y temprana disputa de la cual Sen se nutre. Estudia con Maurice Dobb, el más grande economista marxista inglés, autor de una de las más relevantes síntesis críticas de las teorías en economía: Teorías del valor y de la distribución desde Adam Smith, ideología y teoría económica (Dobb, 1988[1975]). También se manifiesta la presencia de Dennis Robertson, colaborador de Keynes (1920-1930) y de Piero Sraffa; este último protegido de Keynes debido a la persecución sufrida por su relación con Gramsci estando en prisión. Joan Violet Robinson (Robinson, s. f.), merecedora de un premio Nobel, es quien dirige la tesis doctoral de Sen. Seguidora en sus inicios de las teorías de Marshall, transita a 
las teorías de Walras, Keynes y Pigou, interesándose también por las tesis de Marx sobre el valor trabajo. Su recorrido por diferentes países y los múltiples seminarios que ha impartido en los que se reúne una pléyade de intelectuales va a retroalimentar su formación y, al mismo tiempo, a enriquecer el campo.

Estas cuestiones se expresan en una serie de conferencias. Las llamadas Conferencias de Dewey se dieron en Columbia en septiembre de 1984 (Sen A., El bienestar, la condición de ser agente y la libertad, 1998); las de Hennipman en abril de 1982, pronunciadas en Ámsterdam y publicadas con el título de Commodities and Capabilities (Sen A., 1985); las de Tanner en 1985, publicadas bajo el nombre de Standard of Living (Sen, Hart, Kambur, Muelbauer, Williams, \& Hawthorn, 1987); y en las de Roye publicadas como On Ethics and Economics (Sen A., 1987a) (Sen A. , 1989), (Cfr. Salcedo, 1998, págs. 12, nota 10). También edita trabajos sobre la teoría del bienestar y su medición, desarrollo y valores (Sen A. , 1982) (Sen A. , 1984).

\section{Ética, filosofía y economía}

Sin embargo Sen trascendió sus raíces para abrazar simultáneamente la teoría de elección social y la del desarrollo económico quebrando la barrera entre la matematizada "alta teoría” y la economía del "mundo real”. Los campesinos y pobladores rurales que él estudió tienen modalidades de conducta económica que a menudo contradicen los postulados de lo "racional hedonista" que dominaban la teoría económica; en particular, ciertas empresas colectivas durante períodos de sequía contradicen a menudo la racionalidad individual. En esta línea, Sen aplicó las nociones teóricas de juegos y de la elección racional para dar cuenta de tales conductas. Sus contribuciones teóricas han ayudado a entender los mecanismos que explican las hambrunas y la pobreza (Elizalde, 1999) (Sen A. , Desarrollo y libertad, 2000).

Quizás la singularidad más acusada de su teoría es la re-inclusión de temas éticos en asuntos económicos después de la ruptura entre economía y ética que produjo Lionel Robbins tras la publicación de An Essay on the Nature and Significance of Economic Science (Robbins, 1935) y que permeó la economía contemporánea hasta antes de que apareciera Sen (Sen A. , 1989, págs. 21, nº 1).

Los temas éticos se anuncian en obras tempranas, puramente éticas, donde critica por ejemplo la meta-ética de Hare (Salcedo, 1998). También, en la vertiente de la ética del campo, destacará, desde la línea analítica, la presencia de Dworkin y Hare, en varios seminarios y escritos conjuntos. Del primero destaca la ya clásica obra El imperio de la justicia, 1992 y Ética privada e igualitarismo político, 1990 (Dworkin, 1992) (Dworkin, 1990); y, del segundo, el también clásico escrito El concepto del derecho (Hart, 1966). 
Tan importante como esta reposición de la moral en la economía, la presencia preponderante de la filosofía clásica, en la que destacan en primera línea, para nombrar sólo algunos: Aristóteles, Kant, Smith, Condorcet, Smith, Mill, Marx, Pareto. Pero también Sen, por sus "dobles raíces”, ha abierto un diálogo desprejuiciado entre "Occidente y Oriente". La vertiente filosófica del campo, la comparte con muchos intelectuales entre los que destaca Martha Nussbaum, especializada en Aristóteles, -pero también Cohen, Charles Taylor, Michel Walzer-, con la primera comparte la coordinación de uno de los textos más significativos en la construcción del campo y donde se distingue la discusión en torno de una de las categorías más sugerentes referidas al Desarrollo Humano: la calidad de vida (Naussbaum. \& Sen, 1993).

\section{Índice de Desarrollo Humano y organismos internacionales}

Asimismo, es enorme la influencia que Amartya Sen ha ejercido sobre las actuales concepciones sobre el Desarrollo Humano que las $\mathrm{Na}-$ ciones Unidas impulsan a través de sus Informes sobre este tema, poniendo el acento en el Î́ndice de Desarrollo Humano, a nivel global y a nivel de países, y que comenzaron a elaborarse anualmente hace casi ya dos décadas.

Varios son los intelectuales que intervienen junto a Sen en la construcción del campo del Desarrollo Humano.

Mahbub ul Haq fue el coordinador de los primeros informes del PNUD, trabajó como director de Planificación en el Banco Mundial (19701982), ministro de Planificación y Finanzas (1982-1984) fundador de Human Development Centre (Centro para el Desarrollo Humano), en Pakistán, en 1996.

También participa en forma significativa Gro Harlem Brundtland, política noruega (1939), doctora en medicina y master en Salud Pública, miembro del Partido Laborista noruego, ministra de Medio Ambiente en 1974 y Primera Ministra de Noruega en varios periodos; directora general de la Organización Mundial de la Salud (OMS) de 1998 a 2003, es autora del famoso informe que lleva su nombre (Brundtland, 1987). Ella ha sostenido que la violencia era el principal problema de salud pública.

En 1972, Sen fue co-autor con Dasgupta y Marglin, de una famosa guía para la evaluación de proyectos producida por las Naciones Unidas, la cual ha prestado una enorme utilidad a muchísimas organizaciones (Dasgupta, Marglin, \& Sen, 1972).

\section{Algunos alcances al concepto de Desarrollo Humano}

"El desarrollo humano es un proceso mediante el cual se ofrece a las personas mayores oportunidades.” Esta noción está profundamente emparentada con los aportes conceptuales que Sen ha realizado discutien- 
do el concepto de nivel de vida. Para eso ha distinguido los conceptos de "capacidades”, "realizaciones” y "bienes y servicios". "Realizaciones” se refiere a las diversas condiciones de vida (las diversas dimensiones del ser y del hacer) que pueden o no ser alcanzadas, mientras que las "capacidades" se refiere a nuestra habilidad para alcanzar dichas condiciones de vida. "Una realización es un logro mientras que una capacidad es la habilidad para lograr. Las 'realizaciones’ están, en cierto modo, más vinculadas con las condiciones de vida, ya que son diferentes aspectos de las condiciones de vida. Las 'capacidades', por el contrario, son nociones de libertad en el sentido positivo del término: las oportunidades reales que se tienen en relación de la vida que se puede llevar”. Por otra parte Sen rechaza la posesión o acceso a bienes y servicios como el criterio para definir el nivel de vida, argumentando que las tasas de transformación de bienes y servicios a realizaciones varían de persona a persona. Por ejemplo, la situación nutricional de dos personas (realización) puede ser diferente a pesar de que su ingesta alimenticia sea igual. Sen sustituye necesidades por "realizaciones" y capacidades, lo cual le permite rebasar el sentido de "falta de las cosas" que el término necesidades transmite inevitablemente y pasa a una concepción más rica de ser y hacer, de libertad, trascendiendo así las concepciones absolutamente materialistas que respecto a las necesidades humanas han dominado el pensamiento económico reciente (Elizalde, 1999).

\section{La pobreza}

Su trabajo sobre pobreza ha incluido innumerables aproximaciones teóricas notablemente fructíferas en su aplicación. “¿El foco de interés debería ser la pobreza absoluta o la pobreza relativa? ¿Debería estimarse la pobreza como una línea de corte que refleje un nivel debajo del cual la gente está -en cierto sentido- absolutamente empobrecida, o un nivel que refleje los estándares de vida usuales en un determinado país?”. Sen se inclina por la noción de pobreza absoluta, afirmando que: "existe un núcleo irreductible de privación absoluta en nuestra idea de la pobreza, que traduce informes de hambre, desnutrición y sufrimiento visible en un diagnóstico de pobreza sin necesidad de conocer previamente la situación relativa. Por tanto, el enfoque de privación relativa es complementario y no alternativo, del análisis de pobreza en términos de desposesión absoluta”. Sin embargo, reconoce a la vez la subjetividad inherente al tema de la pobreza señalando que: “...el sentimiento de privación de una persona está íntimamente ligado a sus expectativas, a su percepción de lo que es justo y a la noción de quién tiene derecho a disfrutar qué”; y por otra parte que: "Para la persona que estudia y mide la pobreza, las convenciones sociales son hechos ciertos (¿cuáles son los estándares contemporáneos?), y no asuntos de moral o de búsqueda subjetiva (¿Cuáles deberían ser los estándares contemporáneos? ¿Cuáles deberían ser mis valores? ¿Qué siento yo respecto de todo esto?” (ídem). Por lo tanto concluye que a todo aquel que pretende estudiar el fenómeno de la pobreza: "No queda casi ninguna otra alternativa que la de aceptar el ele- 
mento de arbitrariedad presente en la descripción de la pobreza, y en hacer ese elemento tan transparente como sea posible». Esta última afirmación expresa la importancia que para Sen tiene la dimensión ética en la economía como lo manifiesta en "On Ethics and Economics" (Sen A., 1987a) (Elizalde, 1999).

\section{La acción colectiva y el bienestar}

La primera obra que da renombre a Sen se elabora en el marco de la Teoría del bienestar y lleva el título de Choice of Thecniques: An Aspect of the Theory of Planned Economic Development, 1960) (Sen A. , 1969). Adelantándose a su primera gran obra Sen escribe varios artículos que lo consagran definitivamente. Algunos temas se refieren a al ahorro y los sistemas de trabajo y la decisión colectiva: (Sen A. , A possibility Theorem on Mayority Decisions, 1966); (Sen A., Quasitransitivity, Rational Choise and Collective Desicions, 1969); On Optimizing the rate of saving (Sen A., 1961); Labour allocation in a cooperative enterprese (Sen A., 1966), Isolation, assurence and social rate of discount, 1967) (Sen A., 1967), The nature and classes of prescriptive judgements (Sen A., 1967).

Pero la obra que le otorga reconocimiento mundial es Collective Choice and Social Welfare, 1970 (Sen A., 1976). La edición definitiva de este texto se elabora en un famoso Seminario, de enorme significación, para la constitución de campo del Desarrollo Humano, realizado entre 1968 y 1969 con la participación estrecha de Arrow y Rawls.

El problema identificado por Sen a través de esta investigación es el supuesto en la economía de bienestar y de los incomparables beneficios interpersonales. En Elección Colectiva y Bienestar Social, encuentra su clave en el famoso "Teorema de la Imposibilidad de Arrow". Sin esta, Sen arguye, el teorema puede caer; con esta, el teorema es vacío.

Las tesis de Ken Arrow, creador del Teorema sobre la imposibilidad y premio Nobel en economía en 1972, se construyen en el terreno de la Paradoja de Condorcet. Las obras tempranas de Arrow son Social choise and individual Values, 1951 y An extension of the Basic Theorems of Classical Welfare Economics, 1951b) (Arrow, 1974) y son de gran importancia en lo inicios de la constitución del campo de Desarrollo Humano.

Por su parte los postulados de Rawls se elaboran principalmente en dos libros: Liberalismo político, y Teoría de la justicia, (Rawls, 1995[1993]) (Rawls, 2006[1971]). Rawls es sin duda uno de los más influyentes cientistas sociales contemporáneos como es entre muchos Brian Barry que retoma el tema de las teorías de la justicia (Barry, Teorías de la justicia). 


\section{La imposibilidad paretiana}

En la década de los setenta, Sen comienza a desarrollar una conceptualización crítica a los supuestos básicos de la economía que asimila la libertad con eficiencia en las decisiones sociales. Sen rompe con el supuesto del Homo oeconomicus y los estudios tradicionales sobre la pobreza. En este sentido destaca el artículo The Impossibility of a Paretian Liberal (Sen A., 1970) (Sen A., 1986) (Hahn \& Hollis, 1986). El principio paretiano tan importante en algunas concepciones de las ciencias sociales y, especialmente en la economía, jugó un papel preponderante en los argumentos como sobre la imposibilidad (teorema de Arrow). Con Sen este supuesto se convierte en un cuestionamiento de los supuestos fundamentales de la economía hasta ese entonces. No acepta la identificación entre decisiones sociales y eficacia y de ahí parte la elaboración de su futura filosofía.

En La imposibilidad de un Liberal Paretiano argumenta que el supuesto del Optimo de Pareto en la teoría del bienestar no es neutral respecto del valor sino más bien contradice la antigua noción de "liberalismo" de J. S. Mill, ya que el criterio Paretiano no establece salvaguardas para el “espacio personal” (Sen A., 1970) (Sen A., 1986).

En ese sentido desarrolla varios textos referidos a la decisión social y la moralidad y a la crítica al modelo de la decisión como son: Behaviour and the Concept of preference; Choice, Orderngs and Morality; Liberty, Unanimity and Rights; Los tontos racionales: una crítica de los fundamentos conductistas de la teoría económica (Sen A. , 1973) (Sen A. , 1974) (Sen A. , 1976) (Sen A., 1986).

\section{Crecimiento y justicia}

Los temas principales que pone sobre la mesa son la distancia entre la economía del crecimiento y la justicia: disponer de bienes pero no estar en condiciones de gozarlos; la antítesis entre la regulación automática del mercado o el manejo de una dirección dictatorial esclarecida. Se trata de quien decide qué es lo mejor, lo prioritario y la forma de lograrlo para la población. Ambas alternativas no resuelven la injusticia en la distribución de la riqueza; la identificación de la eficiencia del mercado con una cierta libertad sin justicia; la incorporación de equilibrios técnicos en la economía que sustituyeron e ignoraron sus raíces éticas clásicas.

Las revisiones al utilitarismo han sido constantes, entre ellas destaca la controversia sostenida con el premio Nobel de economía John Charles Harsanyi (1920-2000) como lo hace en La polémica Harsanyi-Sen sobre utilitarismo e igualdad (Sen A., 1996). Aún dentro de la teoría de elección social la medición de la desigualdad y los principios de elección social 
igualitaristas siguen siento de su preocupación (Sen A. , Rawls vs. Bentham: An axiomatic Examination of the pure Distribution Problem , 1974). A este campo pertenecen sus conferencias "Radcliffe" pronunciadas en la universidad de Warwick (Sen A., On Economic inequality, 1973) posteriormente es revisada por Sen y Foster en 1997 (Sen A. , La desigualdad económica, 2001).

\section{Hambrunas}

Amartya Sen obtuvo el merecido reconocimiento intelectual de que goza a partir de sus estudios de las hambrunas en Bangladesh, en India y en los países del África Saheliana. Sen trabaja en la economía del desarrollo, el estudio del bienestar de la gente más pobre del mundo actual. Sus más conocidos trabajos desafían la visión común que la escasez de alimento es la más importante explicación de la hambruna. Estudiando varias catástrofes. Sen ha señalado que "las hambrunas han ocurrido aunque la oferta de alimentos no ha sido significativamente más baja que durante los años previos” (sin hambrunas) (ídem).

Parte de su explicación de la hambruna de 1974 en Bangladesh es que las inundaciones que cubrieron el país elevaron los precios de los alimentos, mientras que las oportunidades de trabajo para los trabajadores agrícolas declinaron tanto que una de las cosechas no pudo ser realizada. Debido a estos factores, los ingresos reales de los trabajadores agrícolas declinaron tanto que este grupo fue desproporcionadamente afectado por el hambre. Sen afirma que: "Si una de cada ocho personas sufre regularmente de hambre en el mundo, esto es el resultado de su inhabilidad para alcanzar la titularidad sobre suficientes alimentos; la cuestión de la disponibilidad física de los alimentos no está directamente involucrada”. Proporcionando una explicación a lo anterior, Sen sostiene que: “...la tradición de pensar en términos de lo que existe, más que en términos de quién puede decidir sobre qué. La simpleza fascinante del cociente entre alimentos y población ha sido "obscura" a través de los siglos, y sigue plagando las discusiones de política...” (ídem)

Poverty and Famines en 1981 (Sen A. , 1973), probablemente, es su obra más reconocida. Este trabajo fue el resultado de un estudio preparado para el programa Empleo en el Mundo de la OIT, el cual contribuyó a la Estrategia para el Desarrollo Internacional de las Naciones Unidas -que orientó las políticas contra el desempleo y la pobreza en los años ochenta. En ella se demuestra la tesis que el hambre no es consecuencia de falta de alimentos o falta de recursos, sino de las desigualdades en los mecanismos de distribución de alimentos. Por el contrario es la estructura de derecho de una sociedad la que limita la capacidad de las personas para acceder a los bienes. De tal modo que la privación no siempre se debe a catástrofes naturales sino muchas veces a las estructuras sociales que impiden a las personas hacerse de ellos (Salcedo, 1998, pág. 11). 
En el final de este periodo se destacan publicaciones que indican las direcciones que seguirá su evaluación madura de la teoría del bienestar, entre ellas: (Sen A., Informational Analysis of Moral Pricipes, 1979), (Sen A., Personal Utilities and Public Judgements: or What's Wrong with Welfare Economics?, 1979a), (Sen A., Utilitarianism and Welfarism, 1979b), (Salcedo, 1998, pág. 11).

\section{Teoría de las capacidades}

Entre 1980 y 1988 en Oxford como profesor de Economía Política deja de lado la teoría de Elección Colectiva, aunque mantiene su preocupación por la medición de la pobreza y se aboca a las cuestiones éticas y metaéticas que permiten evaluar el bienestar individual y colectivo mediante el criterio de las "capacidades". Parte importante de la discusión sobre el desarrollo económico fue redefinida a partir de las conferencias de Sen tituladas "Equality of What?", “Bienes y capacidades” y varios artículos (Sen A., 1980), (AA., 1988) (Sen A., 1983), (Sen A., 1982), (Sen A., 1983), (Sen A., 1984). En esta época, se radicaliza la crítica al utilitarismo económico y se traslada hacia el utilitarismo en la filosofía. En esta perspectiva la libertad real de las personas para elegir su modo de vida aparece como en el centro de la evaluación de la justicia de las instituciones sociales.

\section{Conclusión}

Desde 1988 como profesor de Economía y y filosofía en la Universidad de Harvard ha continuado desarrollando sus teorías sobre la pobreza y sobre la concepción de la justicia como libertad en (Sen \& Drèze, Hunger and Public Action, 1989), y (Sen A., Bienestar, justicia y mercado, 1997[1985, 1990, 1993]) (Sen A., The political Economy of Hunger (3 volum.), 1990). En 1988 organizó junto a Marth Naussbaum la primera conferencia sobre calidad de la vida en World Institute for Development Economics Economics Research de La Universidad de las Naciones Unidas en Helsinki publicada como (Sen \& Nussbaum, The Quality of Life, 1993) . Probablemente la obra más relevante de la última época es (Sen A., Inequality Reexamined, 1992), traducido en castellano como (Sen A., Nuevo exámen de la desigualdad, 1995), (cfr., Salcedo, 1998, pág. 13). Otras obras son (Sen A. , Rationality and Freedon , 2002) (Sen A., El nivel de vida, 2001) (Sen A., Nueva economía del Bienestar, 1995) (Sen, Stiglitz, \& Zubero, Se busca trabajo decente, 2007) (Sen A., Sobre la desigualdad económica, 1979) (Sen A., El valor de la democracia, 2006) (Sen A. , How to Judge Globalism, 2002). 
Polis, Revista de la Universidad Bolivariana, Volumen 8, N²3, 2009

\section{Algunos trabajos de Amartya Sen en español}

A. Sen (1969). La selección de técnicas un aspecto de la teoría del desarrollo económico planificado. México: FCE. versidad.

(1976). Elección colectiva y bienestar social. España: Alianza Uni-

(1979). Sobre la desigualdad económica. Editorial crítica.

(1989). Sobre ética y economía. Madrid: Alianza.

(1997[1985, 1990, 1993]). Bienestar, justicia y mercado. Barcelona: Paidós.

(1998). El bienestar, la condición de ser agente y la libertad. En A. Sen, Bienestar, Justicia y mercado (págs. 39-109). Barcelona: Paidos.

(1986). La imposibilidad de un liberal paretiano. En F. Hahn, \& M. (. Hollis, La filosofía y teoría económica . México: FCE.

(1996). La polémica Harsanyi-Sen sobre utilitarismo e igualdad. Telos, $V$, págs. 49-112.

(1986). Los tontos racionales: una crítica de los fundamentos conductistas de la teoría económica. En F. Hahn, \& M. Hollis, Filosofía y teoría económica. México: FCE. Valencia.

(1995). Nueva economía del Bienestar. Valencia: Universidad de

(1995). Nuevo exámen de la desigualdad. Madrid: Alianza.

(2000). Desarrollo y libertad. México: Planeta.

(2001). El nivel de vida. Madrid: Katz.

(2006). El valor de la democracia. Ediciones de intervención electoral.

\section{Algunos trabajos que contribuyen a la construcción del campo}

Arrow, K. J. (1951b). An extension of the Basic Theorems of Classical Welfare Economics. En J. (. Neyman, Procedings of the second Berkeley Symposium of Mathematical Statistics . University of California Press: Berkeley, CA. 
Arrow, K. J. (1951a). Social choise and individual Values. Nueva York: Whiley.

Arrow, K. J. (1974). Tr. cast.: Elección social y valores individuales. Madrid: Instituto de Estudios Fiscales.

Barry, B. (1995[1989]). Teorías de la justicia. Barcelona: Gedisa.

Barry, B. (1995[1989]). Teorías de la justicia. Barcelona: Gedisa.

Dasgupta, ,. P., Marglin, S., \& Sen, A. (1972). Guidelines for Project Evaluation . New York: United Nations.

Dobb, M. (1988[1975]). Teorías del valor y de la distribución desde Adam Smith. ideología y teoría económica. México: Sglo XXI.

Dworkin, R. (1992). El imperio de la justicia. Barcelona: Gedisa.

Dworkin, R. (1990). Ética privada e igualitarismo político. Barcelona: Paidos I.C.E/U.A.B.

Elizalde, A. (1998-1999 de Diciembre-Febrero de 1999). Amarthya Sen: La economía del Bienestar. Plaza Pública , 13-14. co: FCE.

Hahn, F., \& Hollis, M. (. (1986). Filosofía y teoría económica. Méxi-

Hart, H. (1966). El concepto del derecho. (G. R. Carrió, Trad.) Buenos Aires: Abeledo-Perrot.

Keynes, J. M. (1936). The General Theory of Employment, Interest and Money . Cambridge: Harcourt, Brace and Company. FCE.

Naussbaum., M. C., \& Sen, A. (1993). La calidad de vida. México:

Rawls, J. (1995[1993]). Liberalismo político. México: Fondo de Cultura Económica .

Rawls, J. (2006[1971]). Teoría de la justicia. México: Fondo de Cultura Económica.

Robbins, L. (1935). An Essay on the Nature and Significance of Economic Science. Londres: Macmillan.

Robinson, J. (s. f.). Carta de una economista Keynesiana a un economista marxista. México: s. e. 
Salcedo M., D. (1994). Elección social y desigualdad económica. Barcelona: Athropos.

Salcedo, D. (1998). La evaluación de las instituciones sociales según A. K. Sen. En A. K. Sen, Bienestar, justicia y mercado (págs. 9-39). Barcelona: Paidós.

\section{Algunos trabajos de Amartya Sen en inglés}

71

A. Sen (1961). On Optimizing the rate of saving. Economic Journal,

(1966). Labour allocation in a cooperative enterprese . Review of Economics Studies 33 .

34

(1966). A possibility Theorem on Mayority Decisions . Econometrica

(1967). The nature and classes of prescriptive judgements. Philosophical Quarterly, 17 .

(1967). Isolation, assurence and social rate of discount. Quarterly Journal of economics, 81 Sen, A. (1960). Choice of Thecniques: An Aspect of the Theory of Planned Economic Development . Oxford: Oxford.

(1969). Quasi-transitivity, Rational Choise and Collective Desicions. Review of Economic Studies, 36 .

(1970). The Impossibility of a Paretian Liberal. Journal of Political Economy, 78 , págs. 152-157.

(1970). Collective Choice and Social Welfare. San Francisco: Holden-Day.

(1973). Behaviour and the Concept of preference . Economica 40 , 152-157.

(1973). Poverty and Famines: An Essays on Entitlement and Deprivation. Oxford: Oxford Claredon Press.

(1973). On Economic inequality. Oxford: Charenton Press.

(1974). Rawls vs. Bentham: An axiomatic Examination of the pure Distribution Problem . Theory and Decision, 4 , págs. 301-310.

(1974). Choice, Orderngs and Morality. En S. (. Körner, Practical Reason. Oxford: Blackwell. 
(1976). Liberty, Unanimity and Rights. Economica, 43 , 217-245.

(1977). Rational Fools: A critique of Behavioural Foundation of Economic Theory . Philosophy and Publics Affairs, 6 , 317-344.

(1979). Personal Utilities and Public Judgements: or What's Wrong with Welfare Economics? Economic Journal, 89 , 537-558. 463-489.

(1979b). Utilitarianism and Welfarism. Journal of Philosophy, 76 ,

(1979). Informational Analysis of Moral Pricipes. En R. (. Harrison, Rational Action. Cambridge: Cambridge U. P.

(1980). Equality of What? En S. M. McMurrin, The Tanner Lectures on Human Values (vol. I). Salt Lake City: Univ. of Utha Press. 113-132.

(1982). Rights and Agency. Philosophy and Public Affaires, 12 ,

(1982). Choice, Whelfare and Measurement. Oxford and Cambridge, Mass: Blakwell and Mit Press. 28.

(1983). Liberty and Social Choice. Journal of Philosophy, 80 , 5-

(1983). Plural Utility. Procc. of Aristoteliian Society, 81 , 5-28.

(1984). Resourses, Values and Development. Oxford and Cambridge, Mass.: Blackwell and and Harvard University Press .

(1984). Rights and Capabilities. En A. Sen, Resourses, Values and Development. Oxford: Blackwell.

(1985). Commodities and Capabilities. Amsterdam: Amsterdam: North-Holand.

(1986). The Standard of living. En S. MacMurrin, Tanner Lectures on Human Values (pág. Vol. VII). Cambridge: University Press.

(1987). On Ethics and Economics. California: Oxford: Blackwell.

(1990). The political Economy of Hunger (3 volum.) . Oxford: Oxford: Clarendon Press.

(1992). Inequality Reexamined. Oxford: Oxford: Clarenton Press.

(1999). Development as Freedom. N. Y.: Knopf Publishers. 
(2001). La desigualdad económica. México: FCE.

(2002). How to Judge Globalism. The American Prospect, invierno (2002). Rationality and Freedon . Cambridge.

(2007). Identidad y violencia. La ilusión del destino. Argentina: Katz.

Sen, A., Hart, K., Kambur, R., Muelbauer, J., Williams, B., \& Hawthorn, G. (1987). Standard of Living. Cambridge: Cambridge: U. P.

Sen, A., \& Drèze, J. (1989). Hunger and Public Action. Oxford: Oxford: Clarendon Press.

Sen, A., \& Nussbaum, M. (1993). The Quality of Life. Oxford: Oxford: Clarenton Press.

Sen, A., Stiglitz, J. E., \& Zubero, I. (2007). Se busca trabajo decente. Edición Hoac. 